\title{
IgG4-related Disease Presenting as Dural Thickening - A Rare Cause of Myelopathy
}

\author{
J. Zwicker, J. Michaud, C. Torres
}

Can J Neurol Sci. 2014; 41: 392-396

Myelopathy secondary to thickened dura is an unusual clinical presentation which presents considerable diagnostic challenge. The differential diagnosis includes several rare conditions which can only be diagnosed by biopsy. We describe a case of immunoglobulin (Ig)G4-related disease presenting as

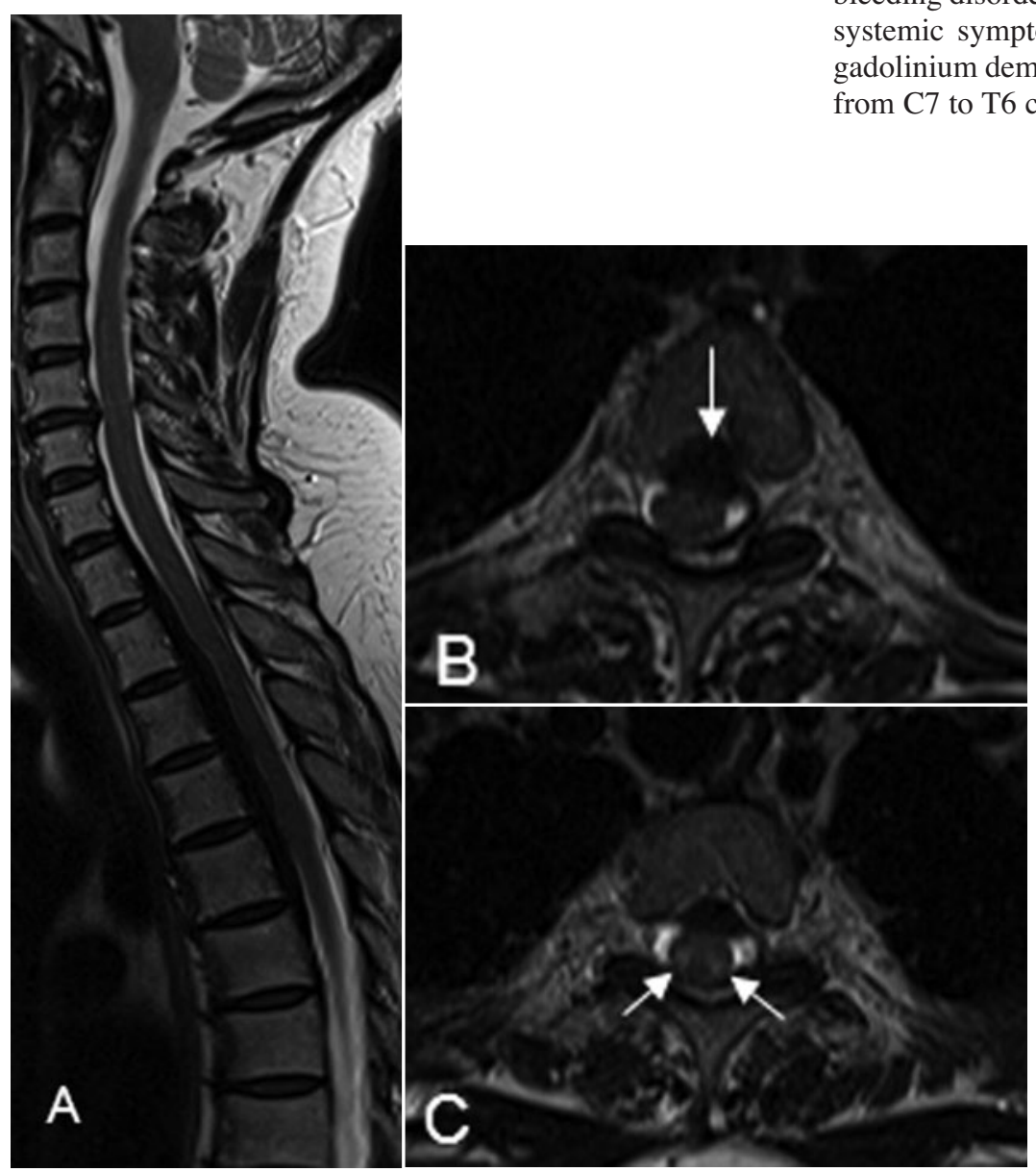

spinal hypertrophic pachymeningitis and review the literature on non-infectious causes of pachymeningitis.

\section{Clinical presentation}

A 58-year-old woman presented with pain in the periscapular region followed by numbness in the feet which extended up the torso over several days. The patient had no history of trauma, or bleeding disorders. She was not anticoagulated. She had no other systemic symptoms. Magnetic resonance imaging (MRI) with gadolinium demonstrated a circumferential epidural-dural lesion from C7 to T6 causing spinal cord compression (Figure 1). This

Figure 1: Sag T2 weighted sequence (1A) of the cervico-thoracic spine shows a dural based hypointense lesion extending from $C 7$ to T6. Axial $T 2 W$ sequence $(1 B)$ at the level of T3 demonstrates marked thickening of the anterior dura (arrow) causing cord compression. Axial T2W image $(1 C)$ at the level of $T 2$ shows increased cord signal (arrows).

\footnotetext{
From the Division of Neurology (JZ), Department of Radiology, Neuroradiology Section (CT), University of Ottawa (JM, JZ), The Ottawa Hospital; Department of Pathology and Laboratory Medicine (JM), Children's Hospital of Eastern Ontario, Ottawa, Ontario, Canada.

Received July 15, 2013. Final Revisions Submitted December 17, 2013.
}

Correspondence to: J. Zwicker, Division of Neurology, The Ottawa Hospital, 1053 Carling Ave, Ottawa, Ontario, K1Y 4E9, Canada. Email: jzwicker@ottawahospital.on.ca. 


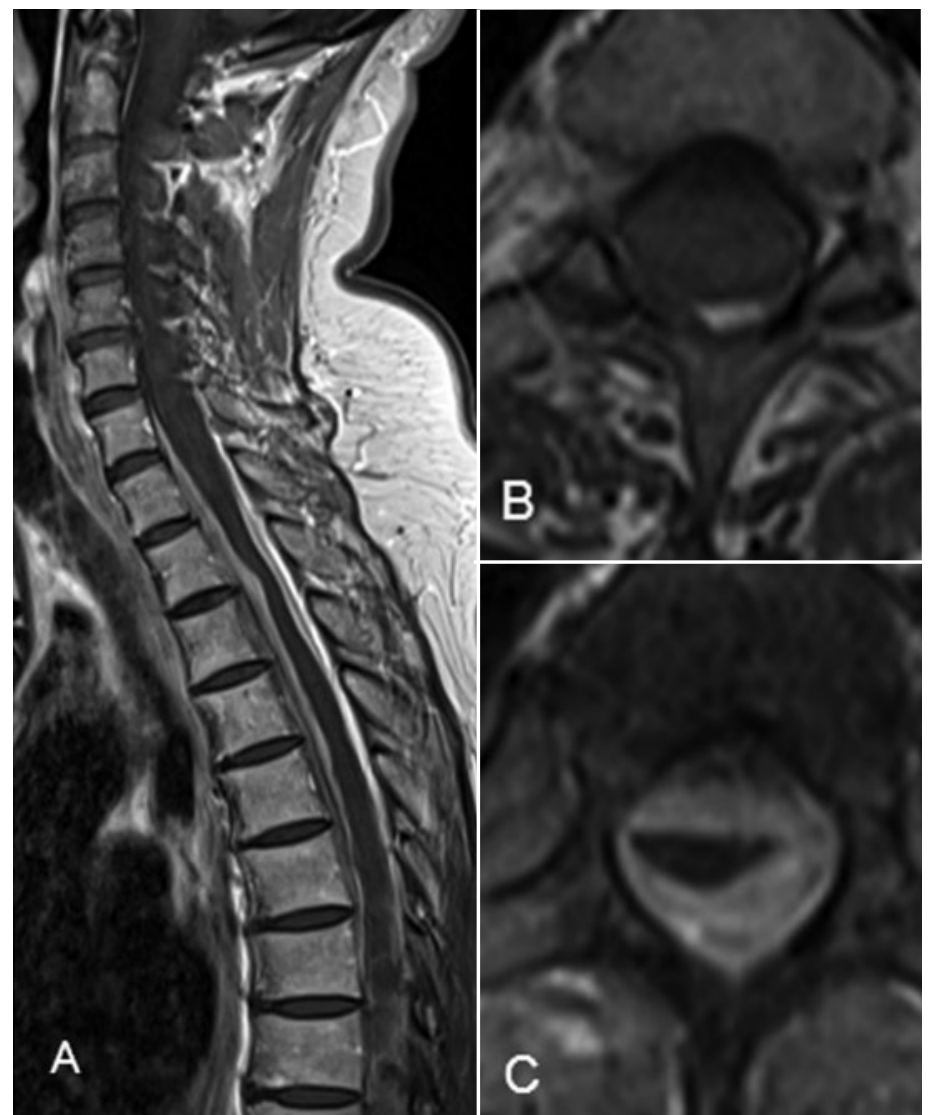

Figure 2: Sagittal T1-weighted sequence post contrast shows thickening and enhancement of the dura from $C 7$ to $T 7$ (A). Axial T1-weighted sequence at T2-T3 demonstrates effacement of the anterior epidural space secondary to hypointense dural thickening (B). Axial T1-weighted sequence with gadolinium at T2-T3 shows marked circumferential thickening and enhancement of the dura, with associated cord compression $(C)$.
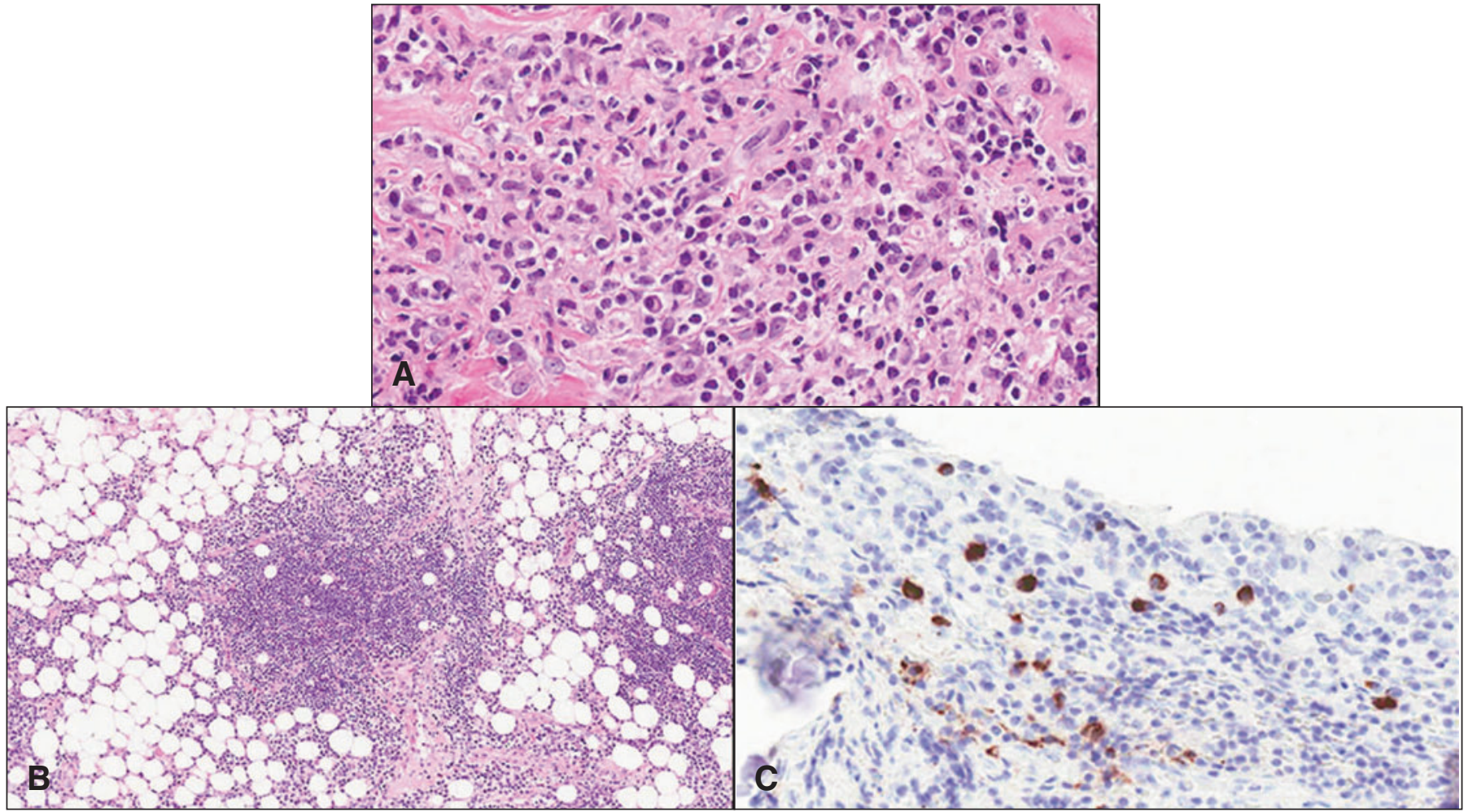

Figure 3: Histology showing (A) a high power view of the dural inflammatory infiltrate composed of lymphocytes, plasma cells and histiocytes (hematoxylin and eosin) (B) a low power view of the inflammatory infiltrate in the adjacent epidural fatty tissue with lymphoplasmacytic follicles and the infiltration between adipocytes (hematoxylin and eosin) and $(C)$ a high power view of IgG4 positive plasma cells in the dura mater (immunoperoxidase technique). 
was felt to represent a dural based process such as inflammatory myofibroblastic tumor, lymphoma or metastases. A computed tomogram (CT) of the chest/abdomen and pelvis demonstrated no neoplasm. Syphilis and tuberculous testing were negative. c-ANCA was positive. Rheumatoid factor was $64 \mathrm{IU} / \mathrm{mL}$ (normal $<14 \mathrm{IU} / \mathrm{mL}$ ). Anti-PR3, p-ANCA, ANA, anti-ENA were negative. Erythrocyte sedimentation rate and C-reactive protein were mildly elevated at $24 \mathrm{~mm} /$ hour (normal 1-20 mm/HR) and $11 \mathrm{mg} / \mathrm{L}$ (normal< $8 \mathrm{mg} / \mathrm{L}$ ) respectively. She had no clinical features of active connective tissue disorder.

At follow-up two months later, the patient's symptoms persisted. There was sustained clonus in the lower extremities and upgoing Babinski responses but no weakness or spasticity. Repeat MRI showed no change. Three months later, the patient presented with weakness of hip flexion and knee flexion and profound spasticity in the lower extremities. The MRI with gadolinium demonstrated circumferential thickening of the dura from C7 toT7 which was hypointense on T1 and T2-weighted imaging and enhanced homogeneously (Figure 2).
T2-T4 laminectomies with excision of the hypertrophic lesion in the dura, and expansion duraplasty was performed on an urgent basis. The epidural tissue was found to be very thickened, fibrosed and adherent to the dura. The dura was $4 \mathrm{~mm}$ thick. The dura was opened in midline and a dissection plane was identified within the dura. This was dissected as far as possible without disturbing the cord. The internal portion was excised.

On histopathology, the dura mater showed an unevenly distributed inflammatory infiltrate (Figure 3). The cellular components of this infiltrate varied from predominantly lymphocytic, lymphoplasmacytic to lymphohistiocytic. The latter was predominant with occasional multinucleated giant cells but without granuloma. Rare scattered eosinophils were also present. Inflammation was present in the wall of a small number of veins but there was no obliteration and otherwise no feature of vasculitis. The inflammation was also present in the epidural fatty tissue, mostly as lymphoid follicles, without germinal centers. Numerous histochemical stains were negative for microorganisms. By immunohistochemistry, polyclonality of

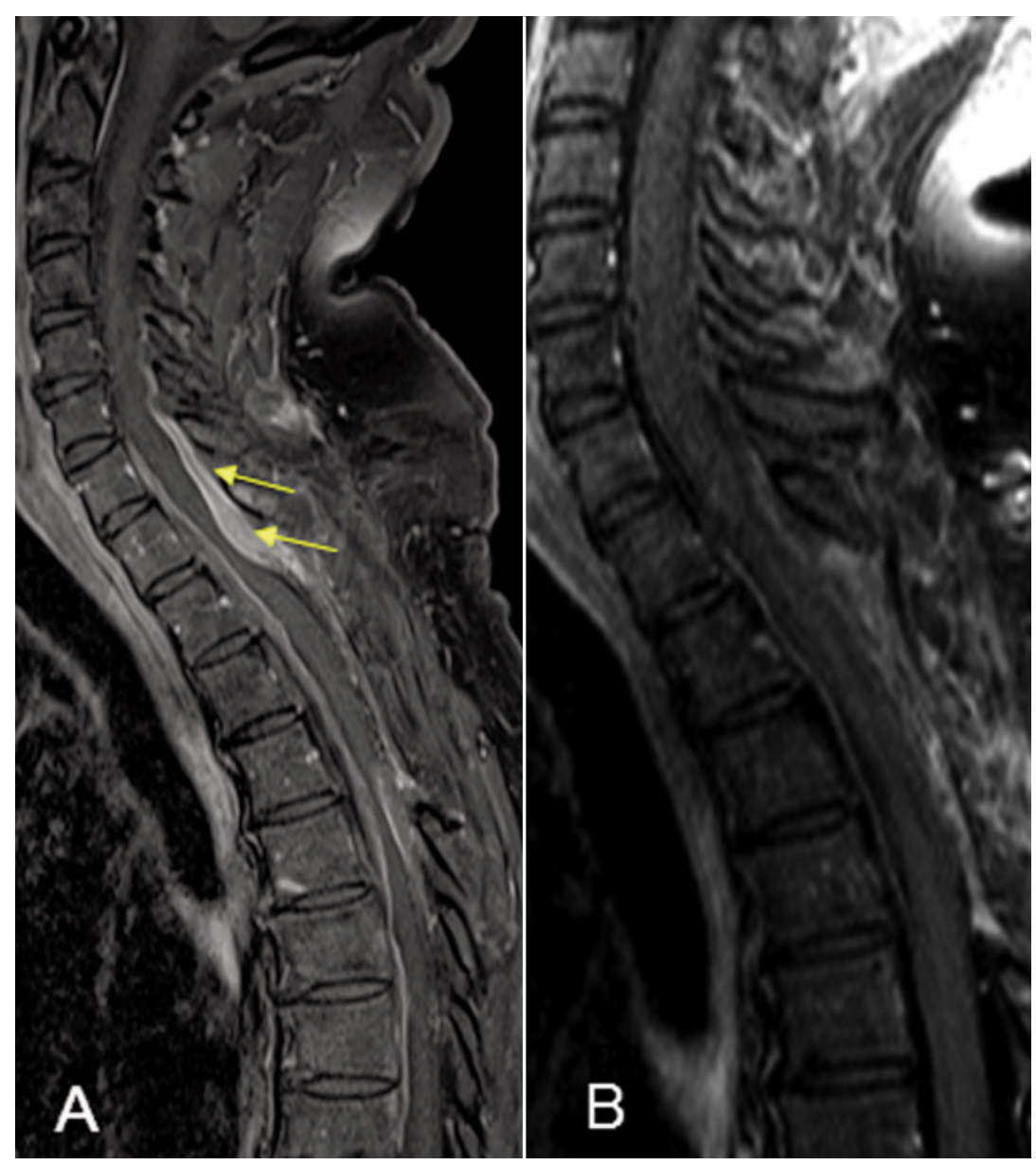

Figure 4: Sagittal T1-weighted sequence post contrast with fat saturation demonstrates marked interval thickening of the dura above the laminectomy level (arrows) with increased cord compression (A). Sagittal T1-weighted sequence post contrast with fat saturation shows significant improvement with decreased dural thickening and resolution of cord compression after treatment with steroids and methotrexate (B). 
the infiltrate was demonstrated with the kappa and lambda antibodies, thus excluding a lymphomatous process, and Langherans cell histiocytosis and inflammatory myofibroblastic tumor were excluded. The findings were consistent with idiopathic hypertrophic pachymeningitis.

The presence of IgG4 positive plasma cells was explored (Figure 3). The average number of IgG4 positive plasma cells was 11.2 per high power field. The IgG4+/IgG+ ratio was $24 \%$. Serum IgG4 was within normal limits.

Post-operatively, the only residual symptom was a feeling of tightness at the costal margin. Ten months postoperatively, she complained of increasing numbness in the legs. Repeat MRI demonstrated progression of the dural thickening causing severe spinal canal stenosis at T5 (Figure 4). She was treated with high dose prednisone and methotrexate. Within days of starting the prednisone her symptoms improved. Four months later the MRI demonstrated significantly improved dural thickening with resolution of the spinal stenosis (Figure 4).

An MRI of the brain with gadolinium done a month later showed no intracranial involvement. At last follow-up, 27 months post-operatively, she was asymptomatic on prednisone and methotrexate aside from some soreness in the legs. The MRI findings have remained stable.

\section{Discussion}

IgG4-related disease is a fibroinflammatory condition characterized by at least two of 1) lymphoplasmacytic infiltration, 2) obliterative phlebitis 3) fibrosis. In addition, increased numbers of IgG4 positive plasma cells and a $\mathrm{IgG} 4+/ \mathrm{IgG}+$ cell ratio of $>40 \%$ is required to be "highly suggestive" of IgG4-related disease as outlined by a recent consensus statement on the pathology of IgG4-related disease ${ }^{1}$. This case represents a "probable" IgG4-related pachymeningitis based on the morphological evaluation with the presence of lymphoplasmacytic inflammation, fibrosis, veinulitis (without obliteration) and increased numbers of IgG4-positive plasma cells ( $\geq 10$ IgG4-positive cells/HPF for meningeal tissue) but without an elevated $\mathrm{IgG} 4+/ \mathrm{IgG}+$ ratio.

Although initially described as a cause of autoimmune pancreatitis, IgG4-related disease has now been reported in almost every organ system. Wallace $e t a l^{2}$ reviewed six cases of idiopathic pachymeningitis and found that $66.6 \%$ of these fulfilled the consensus statement criteria $^{1}$ for IgG4-related disorders. Intracranial mass lesions were seen in two cases, a L5 nerve root mass was seen in one case, and diffuse pachymeningeal thickening alone was seen in one case. Two cases were treated with surgical resection alone. The other two cases responded to prednisone followed by rituximab. Based on their study, Wallace et al suggest that the IgG4-related pachymeningitis may represent a significant subset of idiopathic hypertrophic pachymeningitis .

The term idiopathic hypertrophic pachymeningitis has been used to describe dural-based lymphoplasmacytic meningeal inflammation. It is a rare inflammatory condition which is a diagnosis of exclusion (see Table $1^{3}$ for differential diagnosis). Infectious causes, such as Lyme disease, tuberculosis and syphilis, as well as systemic inflammatory conditions, such as rheumatoid arthritis and Wegener's granulomatosis, must be ruled out clinically and through appropriate testing. Neoplasms, inflammatory myofibroblastic tumor and inflammatory pseudotumor are diagnosed on the basis of histopathological findings.

In this case, the rheumatoid factor was elevated in isolation. Twenty cases of pachymeningitis in rheumatoid arthritis have been previously reported however only two cases were limited to the spinal meninges. One case involved the thoracic region and the other involved the lumbar region. Both spinal cases were treated surgically with marked clinical improvement. The most common histopathological abnormalities of pachymeningitis associated with rheumatoid arthritis are inflammatory cells, and fibrosis. Rarely typical rheumatoid nodules are seen. In patients with central nervous system involvement, improvement with corticosteroids, cyclophosphamide, methotrexate, rituximab or combination therapy has been reported. In all but one case, the patients had manifestations of rheumatoid arthritis prior to the diagnosis of pachymeningitis. Despite the elevated rheumatoid factor, our patient has not had clinical evidence of rheumatoid arthritis in the two years since her presentation for myelopathy.

In idiopathic hypertrophic pachymeningitis (IHP) the clinical presentation depends on whether the cranial dura, spinal dura or both are affected. The most frequent symptoms are headache, cranial neuropathies, and cerebellar ataxia ${ }^{4}$. Spinal IHP is quite rare but presents as back pain and myelopathic symptoms. It typically affects four or more segments and most commonly affects the thoracic region ${ }^{4}$. Treatments of IHP include surgical intervention, such as craniotomy or laminectomy and decompression, and pharmacological treatment with immuno-

Table: Differential diagnosis of thickened, abnormally enhancing, dura matter

\begin{tabular}{l} 
Idiopathic hypertrophic pachymeningitis \\
Intracranial hypotension \\
Infection \\
$\circ \quad$ Lyme disease \\
$\circ \quad$ Syphillis \\
$\circ \quad$ Mycobacterium tuberculosis \\
$\circ \quad$ Fungal infections \\
$\circ \quad$ Cysticercosis \\
$\circ \quad$ HTLV-1 \\
$\circ \quad$ Malignant external necrotizing otitis \\
Systemic autoimmune/vasculitic disorders \\
$\circ \quad$ Wegener granulomatosis \\
$\circ \quad$ Rheumatoid arthritis \\
$\circ \quad$ Sarcoidosis \\
$\circ \quad$ Behcet disease \\
$\circ \quad$ Temporal arteritis \\
$\circ \quad$ Sjogren syndrome \\
$\circ$ Neoplasm \\
$\circ \quad$ Meningioma \\
$\circ \quad$ Lymphoma \\
$\circ \quad$ Inflammatory myofibroblastic tumor/ \\
$\quad$ inflammatorv pseudotumor \\
\hline
\end{tabular}


suppressive medications such as prednisone, azathioprine, methotrexate, or cyclophosphamide. Of ten case reports of patients with spinal IHP, nine were treated with surgery and five were also treated with steroids ${ }^{4}$. One patient was treated only with antibiotics for concurrent infections and died due to respiratory failure. Three patients had a fluctuating course (all were treated with steroids), three patients had symptomatic improvement at 12-18 months (one was treated with steroids), two patients were asymptomatic at follow-up in one year (one was treated with steroids).

On MRI, in IHP the thickened dura is isointense or hypointense on T1-weighted imaging, hypointense on $\mathrm{T} 2$ weighted imaging, and enhances with gadolinium. The enhancement may be peripheral as opposed to homogenous. The dural inflammation may be linear or nodular. Inflammatory myofibroblastic tumor may present with diffuse or localized dural thickening which is isointense on T1-weighted images and hypointense on T2-weighted images and homogeneously enhances. In contrast, chronic subdural hematoma is typically isointense or hypointense on T1-weighted imaging and markedly hyperintense on T2-weighted imaging.

Pathologically, IHP is characterized by lymphocytes, plasma cells, histiocytes, and granulocytes within the dura ${ }^{4}$. It is distinguished from lymphoma by immunohistochemical analysis. It is readily distinguishable from other neoplasms pathologically. Special stains rule out fungi, bacteria, and mycobacteria. It is distinguished pathologically from inflammatory myofibroblastic tumor which is a "distinctive lesion composed of myofibroblastic spindle cells accompanied by an inflammatory infiltrate of plasma cells, lymphocytes, and eosinophils"5. Although inflammatory myofibroblastic tumour (IMT) has been considered synonomous with inflammatory pseudotumor (IP) in the past, they are now considered distinct entities differentiated by clonal rearrangements of the anaplastic lymphoma kinase receptor tyrosine kinase gene and immunohistochemical expression of anaplastic lymphoma kinase protein in IMT5. In addition, antibodies against smooth muscle actin are frequently positive in IMT but not in IP.

The distinguishing clinical characteristics of IMT and IP are not clear, as the two terms have been used interchangeably in the past. Currently, IMT is considered a neoplastic process whereas inflammatory IP is not. In their review of six cases of IMT and 18 cases of IP in the central nervous system, Swain et $a l^{5}$ found that $7 / 18$ pseudotumour patients had concurrently a systemic inflammatory or infectious disorder vs $0 / 6$ with IMT. Two IP patients without systemic disease experienced clinical and radiologic improvement after steroid treatment. None of the patients in the IP group experienced recurrence during the follow-up period (2-143 months). All IMT patients were treated surgically for intracranial mass lesions and only 1/6 IMT patients had a recurrence during the follow-up period (7-123 months).
In summary, prominent dural thickening isolated to the spinal cord may be a manifestation of a systemic infection, a systemic inflammatory process or a neoplasm. Rare cases will be diagnosed pathologically as inflammatory pseudotumor, inflammatory myofibroblastic tumor, or idiopathic hypertrophic pachymeningitis. Recently IgG4-related disease has been reported associated with pachymeningitis. Based on the limited literature to date, inflammatory pseudotumor and inflammatory myofibroblastic tumor appear to be quite responsive to surgical resection alone whereas idiopathic hypertrophic pachymeningitis often requires long term immunomodulating therapy. In the few cases reported thus far, pachymeningits in IgG4-related disease has responded well to steroid treatment. As recognition of these entities improves, the optimal treatment for these conditions may also be refined.

\section{ACKNOWLEDGMENTS}

Evaluation of IgG4 cells was performed courtesy of the Hamilton Health Sciences Center, St-Joseph's Hospital site.

\section{REFERENCES}

1. Deshpande V, Zen Y, Chan JKC, et al. Consensus statement on the pathology of IgG4-related disease. Modern Pathol. 2012;25: 1181-92.

2. Wallace ZS, Carruthers MN, Khosroshahi A, et al. IgG4-related disease and hypertrophic pachymeningitis. Medicine. 2013:92 (4):206-16.

3. Kupersmith MJ, Martin V, Heller G, Shah A, Mitnick HJ. Idiopathic hypertrophic pachymeningitis. Neurology. 2004;62:686-94.

4. Dumont A, Clark A, Sevick R, Myles ST. Idiopathic hypertrophic pachymeningitis: a report of two patients and review of the literature. Can J Neurol Sci. 2000;27:333-40.

5. Swain RS, Tihan T, Horvai A, et al. Inflammatory myofibroblastic tumor of the central nervous system and its relationship to inflammatory pseudotumour. Hum Pathol. 2008;39:410-19. 